\title{
The prevalence of premenstrual syndrome and its relationship with food intake, anxiety and digestive disorders in university student girls
}

\begin{abstract}
Premenstrual syndrome (PMS) including physical, psychological and behavioral symptoms that occur one to two weeks before of the menstrual period, and several factors may play a role in this regard. Therefore, the aim of this study was to investigate the prevalence of premenstrual syndrome and its relationship with anxiety, digestive disorders such as irritable bowel syndrome (IBS) and constipation, as well as food intake in female students. This cross-sectional study was conducted on 200 university student girls resident in dormitories of Ahvaz Jundishapur University of Medical Sciences. General, anxiety, digestive disorder and dietary intake questionnaires were used. For investigation of PMS, a validated questionnaire was used. In this study the prevalence of PMS was $47.5 \%$. There was a significant relationship between PMS and IBS $(\mathrm{p}=0.009)$ and constipation $(\mathrm{p}=0.034)$. Also, it was determined a significant relationship between PMS and obvious anxiety $(\mathrm{p}=0.024)$ and hidden anxiety $(\mathrm{p}=0.001)$. Intake of meats $(\mathrm{p}=0.021)$ and fast foods $(\mathrm{p}=0.002)$ were significantly higher in subjects with PMS symptoms and intake of carbohydrate sources were significantly lower compare with subjects without symptoms $(\mathrm{p}=0.006)$. It was found that there is an association between PMS and anxiety, IBS and food intake. Therefore by presentation appropriate psychological and nutritional consultation, it could be taken a positive step to improve these factors and reduce PMS symptoms among affected girls.
\end{abstract}

Keywords: premenstrual syndrome, anxiety, irritable bowel syndrome, constipation, food intake
Volume 5 Issue 3 - 2017

\author{
Hadi Bazyar,' Fatemeh Mirzaee,' Elahe \\ Akbari-Panah,' Sima Jafarirad ${ }^{2,3}$ \\ 'Student Research Committee, Faculty of Paramedical Sciences, \\ Ahvaz Jundishapur University of Medical Sciences, Iran \\ ${ }^{2}$ Nutrition and Metabolic Diseases Research Center, Ahvaz \\ Jundishapur University of Medical Sciences, , Iran \\ ${ }^{3}$ Department of Nutrition, Faculty of Paramedical Sciences, \\ Ahvaz Jundishapur University of Medical Sciences, Iran
}

Correspondence: Sima Jafarirad, Nutrition and Metabolic Diseases Research Center, Ahvaz Jundishapur University of Medical Sciences, Golestan Boulevard, Ahvaz, Iran, 6I 357-15794, Tel +986 I-337383 I 7, +989 I I 2527976,

Email sjafarirad@gmail.com; jafarirad-s@ajums.ac.ir

Received: November 01, 2017 | Published: December 12, 2017
Abbreviations: PMS, premenstrual syndrome; IBS, irritable bowel syndrome; BMI, body mass index; WC, waist circumference; $\mathrm{HC}$, hip circumference; STAI, spielberg's state-trait anxiety inventory; ACOG, american college of obstetrics and gynecologic disease; SD, standard deviation; FFQ, food frequency questionnaire

\section{Introduction}

Premenstrual syndrome (PMS) involves physical, psychological, and behavioral symptoms that occur one to two weeks before the onset of menstruation in women. The symptoms of this syndrome and their severity often vary between individuals and between successive cycles and reduce to the onset of bleeding. ${ }^{1}$ In most women, there is no relatively predictable pattern for symptoms. ${ }^{2}$ Epidemiological findings suggest that the frequency of PMS in women is more than 80 to $90 \% .^{3}$ Almost $5 \%$ of women have symptoms that are so severe that disrupt their individual and social activities and are called premenstrual dysphoric disorder (PMDD). ${ }^{4,5}$ In a survey in Iran, women 18 to 45years old were studied between 2009 and 2011 and the results showed that the prevalence of PMS was $52.9 \%$, while $34.5 \%$ suffering from severe forms. ${ }^{6}$ Feeling of fragmentation, craving for food, insomnia and/or swallowing, headache, sadness, pelvic pain, breast tenderness, joint pain, and bloating are important symptoms and irritability, anxiety, depression, mood swings, hostility, weak concentration, confusion, social disturbance, and interpersonal conflicts are the common psychological and emotional symptoms of PMS. ${ }^{7,8}$ There is also a number of other PMS symptoms such as constipation, muscle cramps, drowsiness, loss of libido, swelling of the face, forgetfulness, fatigue, headache, herpes outbreaks, hot flashes, light and sound sensation, palpitations, restlessness, weak memory, sore throat, vomiting, and weight gain. ${ }^{9}$ The exact cause of PMS remains unclear and various factors are thought to be involved, therefore it is suggested that PMS be considered as a multi-factor problem. ${ }^{10}$ On the other hand, possible reasons may include hormonal changes, neurotransmitter changes, diet, stress and lifestyle. ${ }^{11}$ Women with PMS suffer from disruption of their social and economic performance, ${ }^{12}$ in addition, their job performance is reduced, thereby, a remarkable decline in economic efficiency will occur. ${ }^{13}$

Different risk factors that affect PMS include high caffeine intake, ${ }^{14}$ stress, age, history of depression, family history, and nutritional factors such as low levels of vitamins and minerals such as vitamin B6, magnesium, ${ }^{15}$ Zinc, manganese, ${ }^{16}$ vitamin $\mathrm{E}$ and vitamin $\mathrm{D}$ as well. Common therapeutic recommendations include medications (antidepressants and anti-anxiety drugs), oral supplements (such as bromocriptine, calcium and magnesium), the consumption of natural products (such as herbal extracts and oils such as primrose oil), lifestyle changes (exercise and diet) and behavioral therapy. ${ }^{17}$

Chronic stress and anxiety are among the external factors that affects PMS. A study showed that higher stress levels are associated with a higher risk of PMDD in women. ${ }^{18}$ In addition, women suffering from PMS experience more stressful situations than healthy people. ${ }^{19,20}$ It has also been reported that women with severe PMDD or PMS have a higher anxiety level than those with mild PMS or no PMS. ${ }^{21}$ It has been shown that $31 \%$ of women with PMS have behavioral disorders, ${ }^{22}$ of which $25 \%$ have anxiety disorder. ${ }^{23,24}$ 
Like PMS, irritable bowel syndrome (IBS) is also a common disorder with psychotic symptoms among these women, ${ }^{25}$ which is a common disorder in the gastrointestinal tract, often associated with diarrhea, constipation, chronic abdominal pain and bloating and covers the majority of the population and has a prevalence of approximately $15-10 \%$ in Europe, North Africa, Australia and other developed countries. The prevalence of IBS in Asia and other developing countries has also increased. ${ }^{26}$ This disorder, like PMS, can significantly reduce the quality of life and increase medical costs. Scientists such as Heitkemper et al., ${ }^{27}$ who examined the effect of gender on IBS showed that women suffered from IBS at higher rates than men and their intestinal symptoms worsen before menstruation. ${ }^{27}$ Other studies also reported that women with IBS who had history of menstrual disorder and PMS had a higher incidence of abdominal pain and discomfort in this area than women with IBS and without PMS. In general, it has been shown that a significant proportion of women with IBS has PMS symptoms or suffered from PMS in the past. ${ }^{28}$

Previous studies reported that nutritional factors may also play an important role in the development of PMS. ${ }^{29,30}$ It has been shown that extra consumption of sweet-tasting food items, fried, fast food, caffeine, alcohol and low intake of vegetables and fruits is significantly associated with the prevalence of PMS. ${ }^{31,32}$ It has been recommended that women with PMS experience, especially those who have a sensitivity to touch the nipple, should refrain from caffeine intake completely. ${ }^{25}$ Since anxiety, IBS and constipation are common factors associated with PMS, the combination of these factors together worsens the physical and psychological symptoms of PMS patients and, due to the that few studies on the relationship between PMS and anxiety, IBS, and constipation has been done, therefore, the aim of this study was to investigate the prevalence of PMS and its association with the level of anxiety, IBS, constipation and diet in girl students.

\section{Materials and methods}

In this cross-sectional study, 200female students resident in dormitories at Ahvaz Jundishapur University of Medical Sciences were selected randomly. This study was approved by the Ethics Committee of Ahvaz Jundishapur University of Medical Sciences (IR AJUMS.REC.1396.218), and all the subjects completed the consent form for participation in the study and all information was kept confidential. Inclusion criteria included age range 18 to 35 , residence in the dormitory, lack of specific treatment for PMS and regular menstruation. Exclusion criteria included pregnancy and lactation, women with irregular menstrual cycle, girls who had a recent medical or psychological problem, those who received any type of hormone replacement therapy, subjects with thyroid dysfunction, polycystic ovaries syndrome and those who did not complete the questionnaire correctly. At the beginning of the work, after completing the informed consent form, a multi-part questionnaire was used to collect the data. This included a demographic and basic information questionnaire [height in centimeters $(\mathrm{cm})$, weight in kilograms, body mass index (BMI) in $\mathrm{kg} / \mathrm{m}^{2}$, waist circumference (WC) $(\mathrm{cm})$, hip circumference $(\mathrm{HC})(\mathrm{cm})$, gender, marital status, ethnicity and history of the disease], the questionnaire for detecting PMS and its severity, the questionnaire of obvious and hidden anxiety symptoms, the IBS symptoms and constipation questionnaire, and the dietary intake questionnaire. In this study, weight was measured using a digital balance scale (Omron, Japan) with a precision of 0.1 kilograms without shoes and with the lowest possible clothes. Height was measured using a tape measurement with a precision of $0.5 \mathrm{~cm}$.
Spielberg's State-Trait Anxiety Inventory (STAI) is widely used in clinical research and practices. The emergence of obvious anxiety is devoted to stressful situations such as talking and discussion, loss of social status, and similar situations. However, hidden anxiety has been shown in the context of individual differences in response to stressful situations. Validity and reliability of this questionnaire have been shown in various studies in Iran. ${ }^{33,34}$

PMS was evaluated using validated Rossignol and Bonlander questionnaire that retrospectively assessed the existence and severity of premenstrual syndrome. To assess the frequency and severity of symptoms of premenstrual syndrome, the American College of obstetrics and Gynecologic Disease (ACOG) diagnostic criteria was used, which is at least one emotional sign and a physical symptom that occurs a few days before menstruation and after beginning of the cycle, its symptoms are discontinued without any medication..$^{35}$ In this study, moderate, severe and very severe grades were considered as PMS and the mild grade was ignored. Irritable bowel syndrome and constipation were determined according to the Rome criteria III. ${ }^{36}$

The dietary intake questionnaire used in this study included 22 food items, indicating the amount of consumption of each food item in the last 3 months. The questionnaire is based on the pyramid of Persian food guide, and the questions include the amount of servings eaten from each subgroup (which is in the main Iranian diet). In general, foods were divided into the following groups in this questionnaire: cereals (rice, pasta, breads), milk and dairy products [milk, yogurt, dough (an Iranian dairy drink), ice cream, cheese and whey], protein and meat products (fish, chicken, calves and sheep, legumes and eggs), fruits, vegetables, fats and oils and miscellaneous foods include a variety of fast food (pizza, sandwich, fried potatoes, falafel, samosa), soft drinks, and Sweets. This questionnaire was prepared and adjusted by Iranian researchers and had appropriate validity and reliability. ${ }^{37}$

\section{Statistical analysis}

Independent t-test was used to compare the quantitative variables between two groups of girls with PMS symptoms and without symptoms and Chi-square test was used to examine the qualitative variables between the two groups. All quantitative data were expressed as mean \pm standard deviation (SD) and qualitative data as percentages. SPSS software version 17.0 was used to analyze the data. $\mathrm{P}<0.05$ was considered as a significant level.

\section{Results and discussion}

The mean age of participants in this study was $23 \pm 2$ years. The findings showed that among 200 participants in the study, 95 subjects had PMS and 105 had no symptoms, therefore the prevalence of PMS in this study was $47.5 \%$ while the highest was observed in the Arab girls and the lowest was found in the Kurdish and Persian ethnicities. On the other hand, $51 \%$ of girls also had a history of PMS. PMS was also reported among married subjects more than others (Table 1). Subjects with PMS differed in terms of anthropometric indices with those without symptoms, as the BMI in these girls was significantly higher than those without symptoms $(\mathrm{p}=0.03)$ (Table 1$)$.

By assessment the levels of obvious and hidden anxiety, the subjects were divided into 5 groups: mild, low- moderate, highmoderate, severe and very severe, with $17 \%, 38 \%, 26 \%, 16 \%$ and $3 \%$ respectively in the obvious anxiety group, and as $13 \%, 48.5 \%$, $26 \%, 10.5 \%$, and $2 \%$ respectively in the hidden anxiety group. Chi-square test showed a significant correlation between PMS with 
obvious anxiety levels $(\mathrm{p}=0.024)$ and hidden anxiety levels $(\mathrm{p}=0.001)$ Also, this test showed a significant correlation between PMS and IBS $(\mathrm{p}=0.009)$ and constipation $(\mathrm{p}=0.034)$ (Table 2). Constipation was $64 \%$ in total for girls and the rate of IBS during menstruation was $73.5 \%$ and this rate was specifically reported in subjects with PMS as $37.5 \%$ and $53.1 \%$ (Table 2).
The results showed that the mean intake protein sources and meats $(\mathrm{p}=0.021)$, as well as fast foods $(\mathrm{p}=0.002)$, were significantly higher in individuals with PMS than without PMS. Also, the mean intake of carbohydrate in these individuals was significantly lower than those without PMS symptoms $(\mathrm{p}=0.006)$. The mean intake in other food groups was not statistically significant (Table 3 ).

Table I Demographic characteristics of individuals with PMS and without symptoms of PMS

\begin{tabular}{|c|c|c|c|c|}
\hline \multirow{2}{*}{\multicolumn{2}{|c|}{ Variable }} & \multicolumn{2}{|l|}{ PMS } & \multirow{2}{*}{ P-Value } \\
\hline & & Yes & No & \\
\hline \multicolumn{2}{|l|}{ Age (years) ${ }^{*}$} & $23.02 \pm 2.19$ & $23.21 \pm 2.01$ & $0.13^{\mathrm{a}}$ \\
\hline \multicolumn{2}{|l|}{ Weight $(\mathrm{kg})^{*}$} & $56.14 \pm 8.27$ & $57.9 \pm 8.39$ & $0.21^{\mathrm{a}}$ \\
\hline \multicolumn{2}{|l|}{ Height $(\mathrm{cm})^{*}$} & $160.16 \pm 5.1$ & $161.13 \pm 5.5$ & $0.5^{\mathrm{a}}$ \\
\hline \multicolumn{2}{|c|}{$\operatorname{BMI}\left(\mathrm{kg} / \mathrm{m}^{2}\right)^{*}$} & $22.56 \pm 3.6$ & $21.62 \pm 2.98$ & $0.03^{\mathrm{a}}$ \\
\hline \multicolumn{2}{|c|}{ Waist to hip circumference $(\mathrm{cm})^{*}$} & $0.76 \pm 0.04$ & $0.75 \pm 0.04$ & $0.5^{\mathrm{a}}$ \\
\hline \multicolumn{2}{|c|}{ Age at first menstruation (years) ${ }^{*}$} & $13.1 \pm 1.46$ & $13.2 \pm 1.42$ & $0.68^{\mathrm{a}}$ \\
\hline \multicolumn{2}{|c|}{ Sleep hours during the 24-hour day* } & $8.24 \pm 1.4$ & $7.97 \pm 1.29$ & $0.15^{\mathrm{a}}$ \\
\hline \multirow{6}{*}{ Ethnicity" } & Arab & $9(75 \%)$ & $3(25 \%)$ & \multirow{6}{*}{$0.143^{\mathrm{b}}$} \\
\hline & Lor & $15(45.5 \%)$ & $18(54.5 \%)$ & \\
\hline & Bakhtiari & $20(45.5 \%)$ & $24(54.5 \%)$ & \\
\hline & Kurdish & $8(40 \%)$ & $12(60 \%)$ & \\
\hline & Turkish & $14(70 \%)$ & $6(30 \%)$ & \\
\hline & Persian & $29(40.85 \%)$ & $42(59.15 \%)$ & \\
\hline \multirow{2}{*}{ Marital $^{\#}$ status } & Married & $13(68.4 \%)$ & $6(31.6 \%)$ & \multirow{2}{*}{$0.24^{\mathrm{b}}$} \\
\hline & Single & $84(46.4 \%)$ & $97(53.6 \%)$ & \\
\hline
\end{tabular}

*The data are expressed as mean \pm standard deviation.

\#Data are expressed in terms of number and percentage.

a: Independent t-test.

b: Chi-square test.

Table 2 Evaluation of the relationship between PMS and anxiety levels and digestive disorders

\begin{tabular}{|c|c|c|c|c|}
\hline \multirow{3}{*}{ Variable } & & \multicolumn{2}{|l|}{ PMS } & \multirow{3}{*}{ P-Value ${ }^{a}$} \\
\hline & & \multirow{2}{*}{$\begin{array}{l}\text { Yes } \\
\text { Number (Percentage) }\end{array}$} & \multirow{2}{*}{$\begin{array}{l}\text { No } \\
\text { Number (Percentage) }\end{array}$} & \\
\hline & & & & \\
\hline \multirow{5}{*}{ Obvious anxiety } & Mild & $9(9.4)$ & $25(23.8)$ & \multirow{5}{*}{0.024} \\
\hline & Low-moderate & $36(37.9)$ & $40(48.1)$ & \\
\hline & High-moderate & $26(27.4)$ & $26(24.8)$ & \\
\hline & Severe & $21(22.1)$ & $11(10.5)$ & \\
\hline & Very severe & $3(3.2)$ & $3(2.9)$ & \\
\hline \multirow{5}{*}{ Hidden anxiety } & Mild & $7(7.4)$ & $19(18.1)$ & \multirow{5}{*}{0.001} \\
\hline & Low-moderate & $41(43.2)$ & $56(53.3)$ & \\
\hline & High-moderate & $32(33.7)$ & 20(19) & \\
\hline & Severe & $15(15.8)$ & $65.7)$ & \\
\hline & Very severe & $0(0)$ & $4(3.8)$ & \\
\hline \multirow[b]{2}{*}{ Constipation } & Yes & $68(53.1)$ & $27(37.5)$ & \multirow[b]{2}{*}{0.034} \\
\hline & No & $60(46.9)$ & $45(62.5)$ & \\
\hline \multirow{3}{*}{ Irritable bowel syndrome } & Yes & $78(53.1)$ & $69(46.9)$ & \multirow{3}{*}{0.009} \\
\hline & & & & \\
\hline & No & $17(32.1)$ & $36(67.9)$ & \\
\hline
\end{tabular}

a, chi- square test. 
Table 3 Comparison of the mean of dietary intake per day in the two groups with and without PMS

\begin{tabular}{llll}
\hline & \multicolumn{2}{l}{ PMS } & \\
\cline { 2 - 3 } Food groups(Serving/Day) & With & Without & \multirow{2}{*}{ P-Value $^{*}$} \\
\cline { 2 - 3 } & Mean \pm SD & Mean \pm SD & \\
\cline { 2 - 3 } Bread and cereals & $7.38 \pm 2.65$ & $8.5 \pm 3.11$ & $0.006^{*}$ \\
Milk and dairy products & $1.94 \pm 1.1$ & $2.04 \pm 1.32$ & 0.58 \\
Animal proteins & $2.33 \pm 0.91$ & $2.02 \pm 0.88$ & $0.017^{*}$ \\
Herbal proteins & $0.25 \pm 0.12$ & $0.26 \pm 0.21$ & 0.65 \\
Vegetables & $0.75 \pm 0.67$ & $0.83 \pm 0.72$ & 0.43 \\
Fruits & $1.65 \pm 1.28$ & $1.48 \pm 1.07$ & 0.3 \\
Oils & $10.13 \pm 2.27$ & $9.45 \pm 2.53$ & 0.08 \\
Sweets & $2.14 \pm 1.36$ & $1.8 \pm 1.33$ & 0.07 \\
Fast foods ${ }^{\sharp}($ Per week) & $1.2 \pm 1.16$ & $0.75 \pm 0.79$ & $0.002^{*}$ \\
\hline
\end{tabular}

*Independent t- test.

The prevalence of PMS in this study was reported $47.5 \%$, which was more prevalent among Arabic and Turkish girls, and less prevalent among Persian girls. The causes of this can be described as heritable backgrounds, different lifestyles and special customs. In a similar study conducted by Badkur D et al. ${ }^{38}$ and colleagues in the female college student in India in 2016, the prevalence of PMS was reported as $39.6 \%$, which was almost near the outcome of this study. ${ }^{38}$

By analyzing BMI in this study, it was found that this index was significantly higher in girls with PMS than in non-symptomatic subjects. Therefore, it can be said that high BMI may play a role in the development and progress of PMS. However, BMI and high anthropometric indices may be the cause of many chronic disorders, which is recommended on the basis of proper weight maintenance. Although the participants in this study had normal anthropometric indices in both with and without PMS symptoms subjects, but even a slight increase in body mass (which could lead to an increase in body fat percentage) can affect the symptoms of this disorder.

The results of this study showed that there is a significant relationship between PMS with IBS and its symptoms as well as constipation. In a case-control study by Zhang et al. ${ }^{26}$ assessed stress levels, menstrual periods, intestinal and psychotic symptoms of reproductive women who have experienced both PMS and IBS; they found that in comparison with patients with IBS alone, stress levels and menstrual disorders were increased in patients with IBS and PMS, and intestinal and psychotic symptoms were more severe and the combination of these diseases prolonged the menstrual period and delayed recovery. ${ }^{26}$ The researchers also found a significant relationship between PMS and constipation, suggesting that constipation may also occur at the same time as PMS, and can lead to stool excretion in subjects with difficulty. One of the possible reasons for this is the diet of individuals. Fruits, vegetables, and legumes are a major source of fiber, and reducing their intake can cause constipation. The results of this study showed that the intake of these foods, especially vegetables, in patients with constipation and PMS was significantly lower than those without PMS symptoms. It should be noted that in the present study, the dietary intake of both groups was lower than the recommended daily intake. The results of this study showed that consumption of high-carbohydrate foods, milk and dairy products, legumes and vegetables in patients with PMS was lower than those without PMS, but the intake of animal protein sources, oils, sweets and fast foods in the group with PMS symptoms was significantly more than those without symptoms. Therefore, it may be argued that carbohydrate sources, milk and dairy products, plant proteins, and vegetables may play a preventative role. In a similar study in 2013, Cheng et al. ${ }^{31}$ showed that consumption sweet foods, fried, fast foods, caffeine, alcohol and low intake of vegetables and fruits are significantly associated with the prevalence of PMS. ${ }^{31}$

Mahmoodi et al. ${ }^{39}$ and colleagues in 2010 showed that the intake of supplements and diets with high-carbohydrate reduced PMS and its symptoms (physical, mental and behavioral) significantly. ${ }^{39}$ Another similar study by Farasati et al. ${ }^{32}$ in 2015 in Iran was carried out to examine the relationship between different food patterns and PMS. The dietary pattern of 320 nurses with and without PMS was measured using the food frequency questionnaire (FFQ) and three Western, traditional and healthy food patterns were investigated using the factor analysis. The findings showed that subjects with a western food pattern experienced more PMS than the two other groups. ${ }^{32}$ The mentioned reason in this study was imbalance between estrogen and progesterone, which can cause PMS, and a low-fat diet with high fiber significantly reduces the level of estrogen phosphate and reduces the symptoms of the disease. ${ }^{40}$ This study showed girls who suffered from PMS symptoms had more anxiety levels. It seems serotonin levels are effective for psychiatric symptoms. High glycemic index carbohydrates increase the plasma ratio of tryptophan to neutral amino acids which lead to increases serotonin levels. ${ }^{32}$ Other possible food-related mechanisms include the role of some vitamins such as B1. This vitamin may reduce PMS symptoms by acting as a coenzyme and affecting the metabolism of carbohydrates, fats, proteins, and in particular amino acids that contribute to the development of PMS physical and psychosomatic symptoms. ${ }^{35}$ Since vegetarian food sources (such as bread and cereals) are one of the good sources of this vitamin, anxiety in patients with PMS may have been due to the lower consumption of carbohydrates in these individuals.

\section{Conclusion}

The results of this study showed that there is a relationship between 
premenstrual syndrome and anxiety, IBS, constipation, and diet. Food intake may have an indirect effect on PMS symptoms, therefore, an appropriate diet, such as increasing the intake of fruits, vegetables, low-fat dairy products, cereals, whole-wheat carbohydrate products, and reduced intake of oils and fast foods may help to reduce the physical and mental problems of these individuals.

\section{Acknowledgements}

This study was financially supported by the Student Research Committee of Ahvaz Jundishapur University of Medical Sciences under the research ethics code IR.AJUMS.REC.1396.218. We would like to thank them along with the female students residing in Ahavz Jundishapur University of Medical Sciences dormitories and everyone else who helped us sincerely throughout this research.

\section{Conflicts of interest}

The author declares no conflict of interest.

\section{References}

1. Nourjah P. Premenstrual syndrome among teacher training university students in Iran. J Obstet Gynaecol India. 2008;58(1):49-52

2. Angst J, Sellaro R, Stolar M, et al. The epidemiology of perimenstrual psychological symptoms. Acta Psychiatr Scand. 2001;104(2):110-116.

3. Dimmock PW, Wyatt KM, Jones PW, et al. Efficacy of selective serotoninreuptake inhibitors in premenstrual syndrome: a systematic review. The lancet. 2000;356(9236):1136-1136.

4. Chocano-Bedoya PO, Manson JE, Hankinson SE, et al. Dietary B vitamin intake and incident premenstrual syndrome. Am J Clin Nut. 2011;93(5):1080-1086.

5. Taylor D. Perimenstrual symptoms and syndromes: Guidelines for symptom management and self-care. Advanced Studies in Medicine. 2005;5(5):228-241.

6. Ramezani Tehra F, Robab Allameh M. Prevalence of premenstrual syndrome and some of its relative factors in reproductive age. The Horizon of Medical Sciences. 2012;18(3):121-127.

7. Vichnin M ,Freeman EW, Lin H, et al. Premenstrual syndrome (PMS) in adolescents: severity and impairment. $J$ Pediatr Adolesc Gynecol. 2006;19(6):397-402.

8. Deuster PA, Adera T, South-Paul J. Biological, social, and behavioral factors associated with premenstrual syndrome. Arch Fam Med. 1999;8(2):122-128.

9. Schilling R. New Treatments for premenstrual syndrome; 2017.

10. Golden RN, Gilmore JH. Serotonin and mood disorders. Psychiatr Ann. 1990;20(10):580-586.

11. Heinemann LA, Minh TD, Heinemann K, et al. Intercountry assessment of the impact of severe premenstrual disorders on work and daily activities. Health Care Women Int. 2012;33(2):109-124.

12. Bailey JW, Cohen LS. Prevalence of mood and anxiety disorders in women who seek treatment for premenstrual syndrome. $J$ Womens Health Gend Based Med. 1999;8(9):1181-1184.

13. Kim DR, Gyulai L, Freeman E, et al. Premenstrual dysphoric disorder and psychiatric co-morbidity. Arch Womens Ment Health. 2004;7(1):37-47.

14. Kaleli S, Aydın Y, Erel CT, et al. Symptomatic treatment of premenstrual mastalgia in premenopausal women with lisuride maleate: a double-blind placebo-controlled randomized study. Fertil Steril. 2001;75(4):718-723.
15. Wyatt KM, Dimmock PW, Jones PW, et al. Efficacy of vitamin B- 6in the treatment of premenstrual syndrome: systematic review. $B M J$. 1999;318(7195):1375-1381.

16. Chuong CJ, Dawson EB. Zinc and copper levels in premenstrual syndrome. Fertil Steril. 1994;62(2):311-320.

17. Kues JN, Janda C, Kleinstauber $M$, et al. Internet-based cognitive behavioural self-help for premenstrual syndrome: study protocol for a randomised controlled trial. Trials. 2014;15:472.

18. Watanabe K, Shirakawa T. Characteristics of perceived stress and salivary levels of secretory immunoglobulin A and cortisol in Japanese women with premenstrual syndrome. Nurs Midwifery stud. 2015;4(2):e24795.

19. Ingram RE. Self-focused attention in clinical disorders: review and a conceptual model. Psychol Bull. 2000;107(2):156-176.

20. Bertone-Johnson ER, Chocano-Bedoya PO, Zagarins SE, et al. Dietary vitamin D intake, 25-hydroxyvitamin D3 levels and premenstrual syndrome in a college-aged population. J Steroid Biochem Mol Biol. 2010;121(1-2):434-437.

21. Bertone-Johnson ER, Hankinson SE, Bendich A, et al. Calcium and vitamin D intake and risk of incident premenstrual syndrome. Arch Intern Med. 2005;165(11):1246-1252.

22. Jahromi BN, Pakmehr S, Hagh-Shenas H. Work stress, premenstrual syndrome and dysphoric disorder: Are there any associations? Iran Red crescent Med J. 2011;13(3):199-202.

23. Fontana AM, Palfai TG. Psychosocial factors in premenstrual dysphoria: stressors, appraisal, and coping processes. $J$ Psychosom Res. 1994;38(6):557-567.

24. Lessa LMM, Chein MB da C, Silva DSM da, et al. Irritable bowel syndrome in women with chronic pelvic pain in a Northeast Brazilian city. Rev Bras Ginicol Obstet. 2013;5(2):84-90.

25. Scanu AM, Bull TJ, Cannas S, et al. Mycobacterium avium subspecies paratuberculosis infection in cases of irritable bowel syndrome and comparison with Crohn's disease and Johne's disease: common neural and immune pathogenicities. J Clin Microbiol. 2007;55(12):3883-3890.

26. Zhang Y, Bo P, Li X. Increased incidence of bowel and psychological symptoms in Chinese female D-IBS patients with premenstrual syndrome. Gastroenterol Nurs. 2014;37(5):351-359.

27. Heitkemper MM, Jarrett ME. Update on irritable bowel syndrome and gender differences. Nutr Clin Pract. 2008;23(3):275-283.

28. Altman G, Cain KC, Motzer S, et al. Increased symptoms in female IBS patients with dysmenorrhea and PMS. Gastroenterol Nurs. 2006;29(1):4 11.

29. Chocano-Bedoya PO, Manson JE, Hankinson SE, et al. Intake of selected minerals and risk of premenstrual syndrome. Am J Epidemiol. 2013;177(10):1118-1127.

30. Pietri P, Vyssoulis G, Vlachopoulos C, et al. Relationship between low-grade inflammation and arterial stiffness in patients with essential hypertension. J Hypertens. 2006;24(11):2231-2238.

31. Cheng SH, Shih CC, Yang YK, et al. Factors associated with premenstrual syndrome-A survey of new female university students. Kaohsiung $J$ Med Sci. 2013;29(2):100-105.

32. Farasati N, Siassi F, Koohdani F, et al. Western dietary pattern is related to premenstrual syndrome: a case-control study. $\mathrm{Br} J$ Nutr. 2015;114(12):2016-2021.

33. Kazemi S, Kazemi S, Ghazimoghaddam K, et al. Music and anxiety in hospitalized children. J Clin Diagn Res. 2012;6(1):94-96. 
34. Roohi Gh, Rahmani H, Abdollahi A, et al. The effect of music on anxiety level and some physiological variables of patients before abdominal surgery. J Gorgan Univ Med Sci. 2005;7(1):75-80.

35. Yonkers KA, Obrien PS, Eriksson E. Premenstrual syndrome. Lancet. 2008;371(9619):1200-1210.

36. Drossman DA. Rome III: the new criteria. Chin J Dig Dis. 2006;7(4):181185.

37. Darabi F, Rasaie N, Jafarirad S. The relationship between premenstrual syndrome and food patterns in university student girls. Jentashapir. $J$ Health Res. 2004;5(6):e26656.
38. Badkur D, Wanjpe A, Singh S, et al. Premenstrual Syndrome among Female Students of Colleges in Ujjain City, Madhya Pradesh. Natl J Community Med. 2016;7(11):878-881.

39. Mahmoodi Z, Shahpoorian F, Bastani F, et al. Comparison of the effect of carbohydrate rich supplement and diet on premenstrual syndrome. $J$ Kermanshah Univ Med Sci. 2010;14(1):10-19.

40. Woods MN, Gorbach SL, Longcope C, et al. Low-fat, high-fiber diet and serum estrone sulfate in premenopausal women. Am J Clin Nutr. 1989;49(6):1179-1183. 